\title{
LA MISIÓN DE LA UNIVERSIDAD Y EL CONOCIMIENTO
}
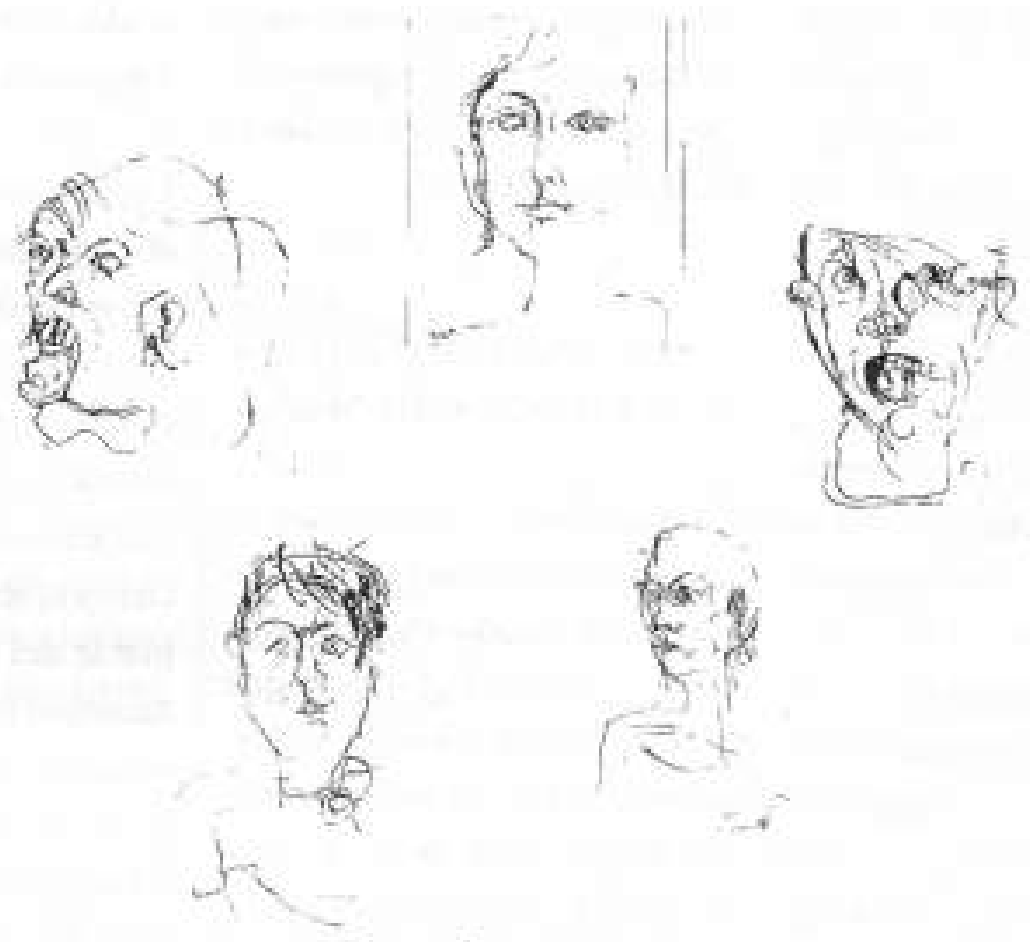

\section{JOSÉ RUBÉN CASTILLO}

Trabajador Sotual.

Magister en Desarrollo Educativo y Social

Profesor Titular Facultad de Fisioterapia

Universidad Autónona de Manizales

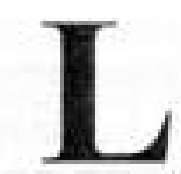

a universidad se ha definido como una institución de indole académica. Este concepto, tiene implicaciones, dado que desde que Platón fundó en Atenas la entidad que denominó $\mathrm{ACADE}-$ MIA. consideró que ésta debia ser una institución de educación superior que se encargaria de una doble función:

"promover la ciencia verdadera y educar una generación de hombres instruidos y preocupados por las cosas públicas que pudiésen salvar a Grecia del naufragio politicon (1).

Son muchas las formas que ha tenido esta propuesta de formaciỏn en y 
para el saber, son variadas las disquisiciones que se han hecho al respecto, tanto en lo conceptual como en la vivencia de quienes se asumen como continuadores de este legado a nivel de educación: pero en lo que se debe estar de acuerdo es en considerar que la labor de la academia tiene que ver directamente con el conocimiento. En otras palabras. la misión de la universidad como ente de educación superior debe involucrar el conocimiento como la base de suacción. Es por ello, que se hace importante en el caso de la Universidad Autónoma de Manizales, que se conciba en su misión como: "una comunidad educadora. dinamizadora del conocimiento..." El asunto está en resolver y adoptar posiciones con relación entre otros a los siguientes interrogantes: ¿Qué es el conocimiento? ¿Qué función cumple? En qué condiciones se produce? ¿Quí caracteristicas tiene?

Un documento comoel presente. es limitado para hacer desarrollos exhaustivos en tomo a esta temática. pero al menos se espera pre- sentar algunos puntos de vista que ayuden a discutirla. Algunos elementos que permiten acercarnos a ello, pueden ser:

\section{EL CONOCIMIENTO ES UNA CALIDAD HUMANA}

En este sentido, no se trata de decir o de sustentar que el conocimientos es exclusivo de nuestra especie. Sin embargo, para que ella se conserve y se desarrolle se requiere de éste. El conocimiento es una necesidad, dado que, para poder subsistir y ejercer en calidad de humano, es a través de él que el hombre puede representarse la realidad, comunicarse con los demás congéneres y aplicarlo para efectos de desenyolverse adecuadamente en el medio. Lo dicho, permite afirmar que un aspecto importante de la calidad de ser humano, es la capacidad que este tiene de utilizar el cerebro para elaborar representaciones acerca de la realidad en la cual ejercen las vidas las personas. para efectos de identificarla, determinar las condiciones en las cuales vive y de orientar adecuadamente su existencia.

Es por esto que, cuando se habla de conocimiento, se debe tener en cuenta que éste es más que la copia de la realidad. por el contrario, el conocimiento implica asumir que éste es el resultado de procesos mentales, mediante los cuales el ser humano busca apropiarse del mundo en que vive. haciendo uso de la capacidad de sentir, representarse. percibir. describir $y$ argumentar dicha realidad. Ello, conduce a la construcción de representaciones, a la abstracción de cualidades $y$ de relaciones que den cuenta de aquellos aspectos que tienen sig. nificado para ellos.

No todo conocimicnto es académico, hay diversidad de formas y niveles, las cuales van desde el cotidiano simple que es imprescindible para la subsistencia del ser humano particular, hasta el más sistemático, propio de comunidades selectivas como en cl caso de las diversas ciencias. 


\section{LA UNIVERSIDADES UNA EMPRESA DEL CO- NOCIMIENTO}

En la universidad se actúa con base en el conocimiento، Los actores de la vida de este tipo de institución son participes de los distintos procesos relacionados con este fenomeno. los cuales van desde la transmisión de las formas de ver el mundo, la apropiación de habilidades especificas de acción y la generación de sistemas de explicación. comprensión y develamiento de la realidad. En este sentido, se favorece tanto la formación de eruditos, de profesionales y de cientificos, bien sea que privilegien el conocimiento existente o la producción del mismo.

Lo anterior, permite ver algunos de los entasis que privilegian la acción en las instituciones universitarias: por un lado forman para el manejo de la información ela. borada por otras fuente al tener como base la transimisión. conel fin de acceder al dominio de saberes. a la apropiación de técnieas. al desarrollo de habilidades y de destrezas. Por otro. dirige su intención al servicio de la construeción del conocimiento, a la preocupación por los procesos $y$ a dar cuenta de las condiciones sociales y culturales en las cuales se produce.

Esto, no impide entender que ambas posiciones pueden coexistir bajo diferentes formas; por el contrario se trata de mostrar que en asuntos de actuar con base en el mancjo, transmisión y producción de conocimiento, sin múltiples las expresiones, niveles, formas, sentidos y énfasis; por ello. es importante asumir posturas frente a la manera tipica de hacerlo. lo cual deviene en la identidad institucional.

\section{ACCION Y PROYECCION DE LA UNIVERSIDAD}

Se da por sentido que ta universidad gestiona y desarrolla su acción a partir del conocimiento. que brinda un espacio para comunidades del saber en cualquiera de sus modalidades; sin embargo. se hace un deber para este tipo de instituciones que se preocupen. entre otros aspectos. por contextuar tanto social como cul. turalmente los contenidos y las formas, es decir. el conocimiento debe ser pertinente para los participantes de los procesos y para la sociedad en la cual funciona.

Otro aspecto digno de tener en cuenta es la variabilidad y la relatividad de los saberes, no se puede desconocer que las verdades del presente son susceptibles de ser reemplazadas por otras. en la medida en que las realidades se modifican y las condiciones cambian.

Además de lo indicado, una de las funciones del conocimientues la de permitir a los seres humanos actuar de manera intencional sobre la realidad, teniendo como base las explicaciones, comprensiones y formas de entender el mundo, buscando con ello, mejorar la condiciòn de vida, tanto individual como colectiva. del ser humano. Es por ello. que el conocimiento tiene sentido en gran parte por la aplicabilidad tanto 
en la acción técnica de modificación del entorno material, como en la manera de orientar los sentidos de vida y de la transformación de situaciones enajenantes.

Estos actos de aplicación del conocimiento sobre el mundo tienen implicaciones, puesto que traen consecuencias tanto en la naturaleza. como en la cultura y en la vida social de las personas. Es por ello, que por encima de la acción. las instituciones universitarias deben preocuparse por asumir posición frente al uso que se haga del conocimiento con le cual trabaja.

En este sentido, también se ve importante que en la Misión de la U. A. M. se plantee qué es una universidad:

"..comprometida con la convivencia pacifica y el desartollo. que contribuve a la formación de personas éticas, con pensamiento critico e innovador".

Esto permite pensar, que si el interés es el de formar personas criticas, reflexivas, de hecho, se asume una posición clara en el sentido de orientar sus esfuerzos tendientes a contribuir a la formación de aprendices más que de aprendedores, en otros términos, se trata de promover acciones en función de formar las estructuras mentales que permitan que los participantes del proceso educativo, desarrollen autonomia de pensamiento y de criterio, puesto que éstas son base para construir criticidad.

Esto en relación con las estructuras de las disciplinas que sirven de respaldo conceptual de la acción de los profesionales. Se trata de preparar las mentes para que puedan discernir acerca de las condiciones que le presenta la vida, más que en el manejo instrumental de los conocimientos. que logren combinar adecuadamente los procesos para atender las demandas prácticas de la realidad, para intervenirla y transformarla, asi como dar la posibilidad de entender los sentidos que le ofrece la vida a los seres humanos. Para ello se requiere lograr competencias que le permitan al estudiante acceder a los asuntos genéricos. de contexto, de principios y de estructuras.

Esto nos genera entre muchas otras inquietudes. la necesidad de penetrar en los procesos que permiten construir los conocimientos. en términos de buscar la lógica de construcción de los mismos, a partir de las operaciones mentales y funcionales que favorecen y permiten la construcción de conceptos que están en la base de la producción de los conocimientos.

En otros términos, se trata de abordar los acontecimientos. sucesos y procesos que ocurren en el pensamiento, para efectos de construir conocimientos. Este es un asunto que debe acompañar el saber de los docentes, en tanto hace parte de los criterios pedagógicos que sirven de base a su desempeño, siempre y cuando se preocupe por contribuir a los procesos de formación de pensamiento reflexivo. critico, autònomo y creador de conocimientos. \% 